\title{
Space interferometry application of laser frequency stabilization with molecular iodine
}

\author{
Volker Leonhardt, Jordan Camp \\ NASA Goddard Space Flight Center, Code 663, Greenbelt, MD 207r1 \\ volker@milkyway.gsfc.nasa.gov
}

\begin{abstract}
A number of planned space interferometry missions, including the LISA gravitational wave detector, require a laser system with high frequency stability over long timescales. A $1064 \mathrm{~nm}$ wavelength NPRO laser stabilized to a resonant transition in molecular iodine is suitable for these missions, providing high frequency stability at an absolute reference frequency. The iodine stabilized laser also offers low sensitivity to temperature and alignment fluctuations, and allows frequency tuning. We have evaluated the noise performance of an NRPO laser stabilized to iodine using frequency modulation spectroscopy, and find an Allan standard deviation of $10^{-14}$ over $100 \mathrm{~s}$. Simplified optical configurations and the radiation hardness of the frequency doubling crystals have also been investigated. (c) 2005 Optical Society of America
\end{abstract}

OCIS codes: $300.6380,350.6090$

\section{Introduction}

High precision length measurements will play an increasing role in future space missions. Among those missions are the LISA gravitational wave detector, ${ }^{1}$ the TPF-C terrestrial planet finder ${ }^{2}$ and the MAXIM x-ray interferometer. ${ }^{3}$ These missions require either highly accurate measurements of changes in the separation of different spacecraft, or high precision spatial stabilization of the optics within one spacecraft. The spatial accuracy needed for these missions requires laser interferometry.

The minimum length change $\Delta L$ that can be detected by a length measurement over the distance $L$ in a linear configuration depends on both the frequency $\nu$ of the laser and on the frequency noise $\triangle \nu$ :

$$
\frac{\triangle L}{L}=\frac{\triangle \nu}{\nu}
$$


The TPF-C telescope requires a mirror separation stability of $25 \mathrm{~nm}$ over a distance of $12 \mathrm{~m}$ for timescales of 8 hours, ${ }^{4}$ while the MAXIM interferometer optics require mirror separation stabilities of less than $1 \mathrm{~nm}$ at distances of a centimeter, ${ }^{3}$ also over 8 hours. With a factor of 10 safety margin we obtain a fractional frequency stability requirement of $10^{-10}$ for TPF-C and $10^{-8}$ for MAXIM. For the LISA mission the frequency noise requirement is given as a spectral shape, $30 * \sqrt{1+\left(10^{-3} / f\right)^{4}} \mathrm{~Hz} / \sqrt{\mathrm{Hz}}$ between $10^{-4} \mathrm{mHz}$ and $1 \mathrm{~Hz} \cdot{ }^{5}$ Non planar ring oscillator lasers at a wavelength of $1064 \mathrm{~nm}$ are known to provide excellent spatial, amplitude and frequency stability. However for the missions referred to above the frequency stability of the laser, roughly $10^{4} / f \mathrm{~Hz} / \sqrt{\mathrm{Hz}}$ with a drift up to $50 \mathrm{MHz} /$ hour, is not sufficient. Much higher frequency stability can be achieved by locking the laser to an external frequency reference.

An extensively used example of such a reference is an optical resonator cavity made of a material with a low thermal expansion coefficient. The length $\mathrm{L}$ of the cavity defines resonance frequencies $\nu_{n}=\mathrm{nc} / 2 \mathrm{~L}$, where $\mathrm{c}$ is the speed of light and $\mathrm{n}$ is an integer, which can be used to stabilize the laser. However, because many frequencies can satisfy the resonance condition, a cavity does not provide an absolute frequency reference. In addition, over long timescales cavities can show large thermal drifts in response to temperature changes of the environment, and furthermore, because of materials effects at sub-angstrom length scales, even at constant temperature the cavity length may not be stable. ${ }^{6}$

In contrast, a molecular resonance provides an absolute frequency reference with a much lower frequency variation with temperature. Molecular iodine is often used for frequency stabilization because it provides strong narrow resonances at $532 \mathrm{~nm}$ that can be accessed by frequency doubling a fraction of the $1064 \mathrm{~nm}$ laser light. Because of these advantages we chose to investigate the use of iodine frequency stabilization for space interferometry applications.

A variety of optical configurations can be used for laser frequency stabilization to Doppler free iodine resonances. In the past excellent frequency stability has been achieved with the technique of modulation transfer spectroscopy. ${ }^{7,8}$ In this work we have used the technique of frequency modulation spectroscopy. ${ }^{9}$ Simpler locking schemes that require no modulators have been implemented in the past with excellent sensitivity ${ }^{10}$ and have also been investigated by us.

In section 2 we describe our experimental set-up, and in section 3 we show the frequency noise results that were achieved. In section 4 we consider the issues of the space application of the iodine system, including the sensitivity of the system to alignment and temperature disturbances, alternative simplified optical configurations, and the radiation hardness of the frequency doubling crystal. In Section 5 the results are summarized. 


\section{Experimental layout}

Our configuration (frequency modulation spectroscopy) uses a pump beam directed through the iodine cell to saturate the cell absorption, and a probe beam to detect the laser frequency deviation from the center of the resonance. The pump and probe beam are set to counterpropagate through the cell, selecting out molecules of a fixed velocity, thus providing a Doppler-free resonance. The pump beam is chopped with an acousto-optic modulator (AOM) to remove the broad absorption profile, while the probe beam is RF modulated with an electo-optic modulator (EOM) to give a linear error signal.

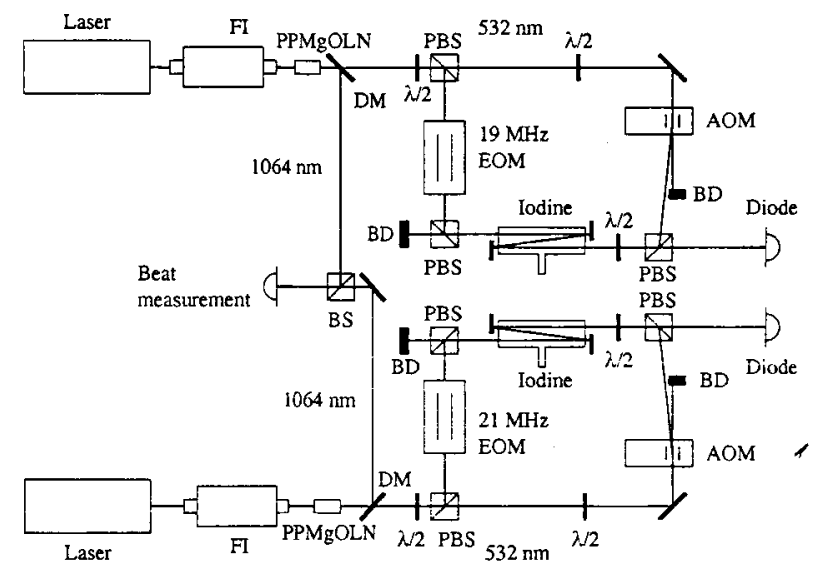

Fig. 1. Layout of the two iodine stabilized laser systems: FI, Faraday isolator; PBS, polarizing beam splitter; BS, non polarizing beam splitter; BD, beam dump; DM, dichroic mirror; EOM, electro optic modulator; AOM, acousto optic modulater; PPMgOLN, periodically poled magnesium oxide doped lithium niobate crystal.

The detailed setup consists of two nearly identical stabilized laser systems (See Fig. 1). The $1064 \mathrm{~nm}$ (infrared) laser light first passes a periodically poled magnesium oxide doped lithium niobate (PPMgOLN) crystal, kept at a temperature of $53.5^{\circ} \mathrm{C}$. The crystal doubles the frequency of a small fraction of the infrared light to $532 \mathrm{~nm}$ (green) light. After separation from the infrared light, the green light is split into a pump beam and a probe beam by a polarizing beam splitter. The power ratio between the beams can be adjusted, the best performance was achieved with roughly equal power of $1 \mathrm{~mW}$ in both beams. The pump beam is passed through an AOM where the beam is chopped at a frequency of $9 \mathrm{kHz}$ for one laser system and at $9.9 \mathrm{kHz}$ for the other. The AOM also increases the pump beam frequency by its driver frequency of $80 \mathrm{MHz}$, while the pump frequency in the other system is reduced by $80 \mathrm{MHz}$. After the AOM the pump light passes the $20 \mathrm{~cm}$ long iodine cell. The probe beam 
is sent through an EOM where the light is modulated at $19 \mathrm{MHz}$ and $21 \mathrm{MHz}$ for the two systems. The probe beam then passes the iodine cell where it is overlapped with the pump beam. The pressure in the iodine cell is kept constant at $\sim 2.3 \mathrm{~Pa}\left(-5^{\circ} \mathrm{C}\right.$ with fluctuations of $\sim 0.1^{\circ} \mathrm{C}$ ) over 15 hours by cooling and stabilizing the cold finger of the iodine cell. The beam diameters for pump and probe beams are $1 \mathrm{~mm}$. Both beams are triple passed through the iodine cell to increase its effective length to $60 \mathrm{~cm}$.

After the cells the beams are extracted by using a halfwave plate and polarizing beam splitters. The pump beam is detected by a photodiode and the signal is demodulated first at the EOM modulation frequency by a mixer. The signal is then demodulated by a lock in amplifier at the AOM chopping frequency. By feeding back the resulting signal the laser is locked to a Doppler free iodine resonance. The temperature of the laser crystal is used as an actuator at low frequencies and a piezo on the laser crystal provides actuation at higher frequencies. The locking bandwidth is typically above $100 \mathrm{~Hz}$. For this configuration we locked to the $R(56) 32-0$ al resonance. ${ }^{7,11}$

A portion of the infrared light is used for a beat measurement with light from the second laser system. With the pump beams shifted by $80 \mathrm{MHz}$ in different directions and unshifted probe beams, the resulting beat frequency for the infrared light is $40 \mathrm{MHz}$. The sum of the frequency noise of the two systems is measured by monitoring the stability of the $40 \mathrm{MHz}$ beat frequency.

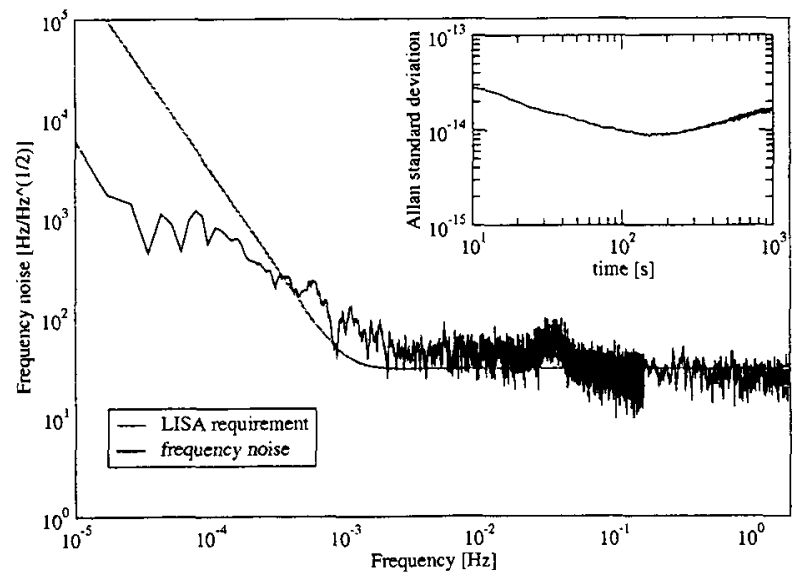

Fig. 2. Linear spectral density of the frequency noise of the two iodine systems, as well as the mission requirement for LISA. The corresponding Allan standard deviation is also shown. 


\section{Frequency noise results}

The resulting linear spectral density and the Allan standard deviation are shown in Fig. 2. The frequency noise falls as $1 / \mathrm{f}$ at low frequencies and becomes almost flat above $3 \mathrm{mHz}$. Thermal drifts in the EOM at low frequencies and amplitude fluctuations of the green light at higher frequencies appear to limit the achieved sensitivity. To our knowledge these are the best results achieved for the frequency modulation configuration. The frequency noise requirement for LISA is also shown. A maximum relative drift of the two iodine systems of $300 \mathrm{~Hz}$ over 15 hours was observed (with a room temperature change of about $2^{\circ} \mathrm{C}$ ). This easily meets the frequency stability requirements for TPF-C and MAXIM.

To exclude the possibility of the frequency noise measurements being artificially low due to the presence of common mode frequency noise of the two iodine systems, beat measurements were taken between one of the iodine systems and a separate laser system stabilized to a thermally shielded ULE cavity in a vacuum tank. [reference Guidos note here] The cavity had a frequency noise about a factor of two lower than the iodine system between $10^{-4} \mathrm{mHz}$ and $0.1 \mathrm{~Hz}$. No additional noise was observed in the cavity iodine beat measurement, indicating the lack of common mode noise in the iodine systems at those frequencies.

\section{Space application of the iodine system}

A critical issue in the implementation of an iodine system in space is the system robustness and reliability. With this in mind we have investigated the sensitivity of the iodine system to alignment and temperature variations, considered the lock acquisition of the system, and have also examined the noise performance of simplified optical configurations. We have also looked at the radiation hardness of the frequency doubling crystals.

A relatively large misalignment of $5 \mathrm{mrad}$ of the light traversing the doubling crystal will result in a drop of the conversion efficiency of only $10 \%$. At the iodine cell a misalignment of the light passing through the cells results in a smaller beam overlap region and thus a smaller effective cell length. We found a 4 mrad misalignment to result in a factor of 10 higher frequency noise (See Fig. 3). Also shown is a measurement without temperature stabilization of the iodine cell cold finger while still cooling it. Its temperature drifted by $1^{\circ} \mathrm{C}$ over 15 hours, but no additional frequency noise was observed between $10^{-4} \mathrm{mHz}$ and $0.1 \mathrm{~Hz}$. The frequency drift of one unstabilized system was less than $1 \mathrm{kHz}$.

To acquire lock of the iodine system in space the laser frequency has to be tuned until the appropriate iodine resonance is accessed. Because the series of iodine resonances have a distinct, well known structure, recognition of the proper resonance is expected to be straightforward. A further issue is the phase locking of two or more stabilized laser systems together. This is an important consideration for missions such as LISA, where independent laser systems on separate spacecraft must operate at frequencies sufficiently close that they 


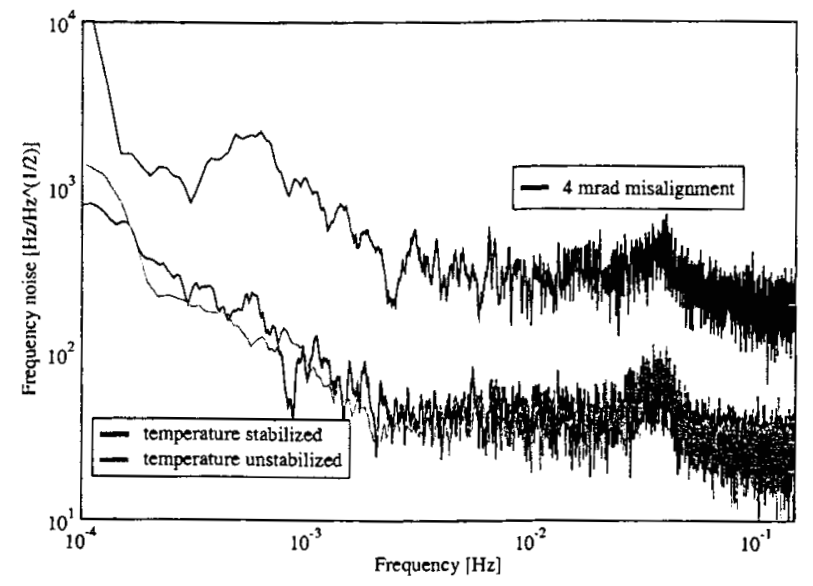

Fig. 3. Frequency noise with beams misaligned by $4 \mathrm{mrad}$ at the iodine cell and noise with and without iodine temperature stabilization

can be phase locked. (Generally this means the beat frequency between the two systems must be within the bandwidth of a high sensitivity photodiode, typically $\sim 100 \mathrm{MHz}$ ). A crucial advantage of the iodine system is the existence of an absolute reference frequency. Our measurements show that lasers locked to the same iodine resonance will run at the same frequency within one or two $\mathrm{kHz}$, allowing acquisition of phase locking. If a frequency actuation of one of the systems should still be required to counter, for example, a Doppler shift of the laser frequencies due to relative motion of the spacecrafts, the AOM driver frequency can be changed. (The simple refinement of optically double passing the AOM would avoid pointing variation as the frequency is shifted, as well as double the frequency shift for a given driver shift.)

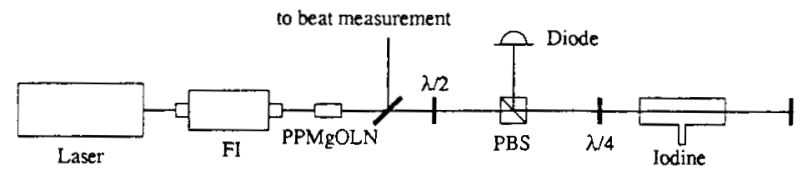

Fig. 4. Simplified optical set-up without modulators.

Another important aspect of system robustness in space is design simplification. With the goal of developing configurations using a minimum number of sensitive electro-optical components, we have investigated simplified setups which still offer good frequency noise performance. A set-up without AOM or EOM modulators is shown in Fig. 4. It uses just one beam backreflected on itself to provide Doppler-free error signals. In this scheme the 
laser piezo is used as the frequency modulator. A frequency modulation amplitude of $1 \mathrm{MHz}$ (approximately the width of the iodine resonance) at a modulation frequency of $20 \mathrm{kHz}$ was used to lock the laser. The double resonance $R(57) 32-0$ a $5+a 6$ produced the best error signals. Demodulating the error signal at the first RF harmonic led to a frequency noise sensitivity to optical alignment due to stray light reaching the photodiode. Demodulation at the third harmonic reduced the sensitivity to alignment because stray light that has not passed the iodine cell does not carry any contribution at the third harmonic. The sensitivity is shown in Fig. 5.

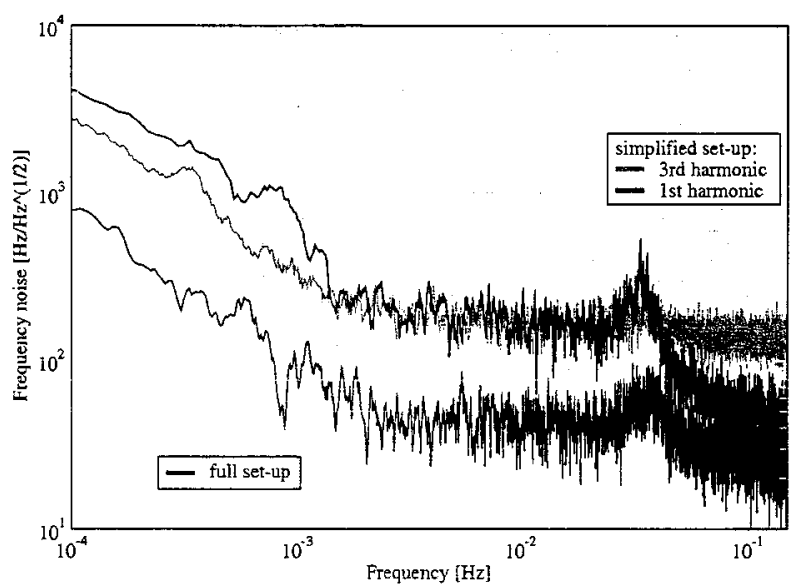

Fig. 5. Frequency noise in the simplified configuration (no EOM, no AOM) with first harmonic demodulation and third harmonic demodulation. The noise of the full frequency modulation spectroscopy set-up is also shown.

A consequence of the use of the laser piezo as the frequency modulator is the appearance of the modulation frequency on the full power infrared light. An unmodulated infrared beam can be produced if an EOM is included after the wavelength separation. We found a factor of 2 to 3 higher noise relative to a modulated laser, due to the smaller size of the frequency modulation produced by the EOM compared to the laser piezo.

The application of the iodine system to the space environment also involves considering the effects of radiation on the system components. The most important issue is the effect of radiation exposure on the efficiency of the frequency doubling crystals. ${ }^{12}$ (Many of the other components have already been space qualified, with the exception of the NPRO laser, which we do not consider here, and the iodine reference cell, a simple sealed quartz tube which is not expected to be adversely affected by radiation.) We used the calculated radiative flux of the LISA orbital environment as our target level. ${ }^{13}$ The 10 year LISA radiation dose is $\sim 8 * 10^{10}$ protons $/ \mathrm{cm}^{2}$ with $90 \%$ of the flux below $70 \mathrm{MeV}$ (assuming 7 solar active years 
and $2.5 \mathrm{~mm}$ of aluminum shielding). Similar radiation levels can be expected for TPF-C and MAXIM as their orbits will also be in deep space (far from the earth radiation belts). We exposed two PPMgOLN crystals, and also one periodically poled potassium niobate (PPKTP) crystal to a proton radiation level of $10^{11}$ protons $/ \mathrm{cm}^{2}$ at $70 \mathrm{MeV}$. In addition to the LISA radiation environment, the PPKTP crystal and one of the PPMgOLN crystals were exposed to increasing doses of gamma radiation to examine the sensitivity of the crystals to its different damage mechanism. The radiation started with $25 \mathrm{krad}_{S i}$ and was increased to a total dose of $60 \mathrm{krad}, 200 \mathrm{krad}$ and then $600 \mathrm{krad}$. Finally all three crystals were exposed to $10^{12}$ protons $/ \mathrm{cm}^{2}$ at $63 \mathrm{MeV}$. No significant effect was observed for the proton and gamma radiation (see Fig. 6). (The gamma radiation did cause a temporary drop of up to $20 \%$ in second harmonic generation efficiency of the PPKTP, as well as a $50 \%$ decrease in green light transmission through the crystal. However the crystal was healed within minutes by application of the $600 \mathrm{~mW}$ infrared beam. Neither drop was observed for the PPMgOLN crystal.) From our results we conclude that the frequency doubling crystals survive radiation levels beyond the anticipated LISA 10 year radiation dose with negligible change in performance.

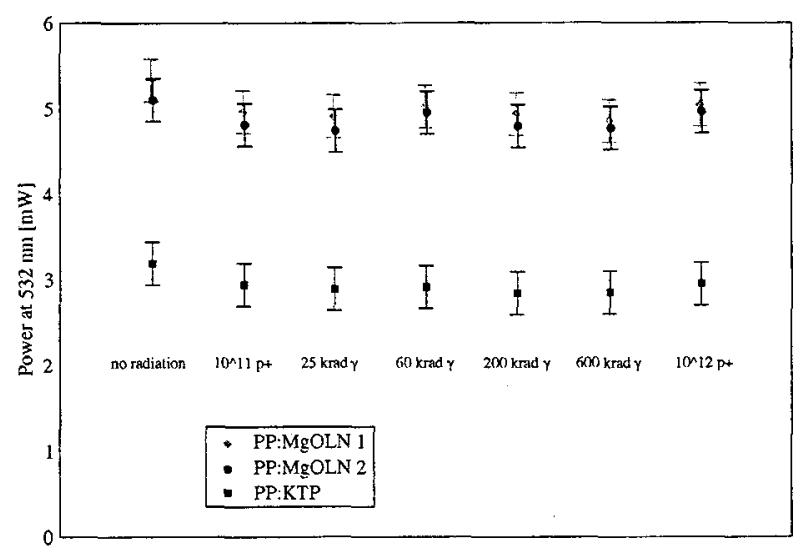

Fig. 6. Light power at $532 \mathrm{~nm}$ generated in the doubling crystal before radiation, after $10^{11}$ protons $/ \mathrm{cm}^{2}, 25 \mathrm{krad}$ gamma radiation, $60 \mathrm{krad}, 200 \mathrm{krad}$, $600 \mathrm{krad}$ and $10^{12}$ protons $/ \mathrm{cm}^{2}$. Crystal 1 was not exposed to gamma radiation

\section{Summary}

We have stabilized two NPRO lasers to molecular iodine using frequency modulation spectroscopy and evaluated the frequency noise. We have achieved an Allan standard deviation of $10^{-14}$ over $100 \mathrm{~s}$, which is sufficient for the space interferometry applications discussed earlier. The iodine system was found to show a low sensitivity to thermal distortions and 
to misalignment. Iodine provides an absolute frequency reference (important for missions requiring phase locking of lasers in separate spacecraft) and the straightforward implementation of frequency actuation (important for missions needing to compensate Doppler shifts due to spacecraft motion). Simplified configurations that involve fewer optical components with a factor of 10 increase in frequency noise have also been investigated. Finally, radiation exposure tests indicate that the efficiency of the frequency doubling crystals will not be degraded in the LISA radiation environment. Due to the obtained results we believe that iodine stabilized laser systems show great potential for future space interferometry applications.

\section{Acknowledgments}

We want to acknowledge Paul McNamaras work on the thermally shielded cavities. We also thank Anthony Martino and ... for their comments on this manuscript.

\section{References}

1. K. Danzmann, "LISA mission overview," Adv. in Space Res. 25, 1129-1136 (2000)

2. M. J. Kuchner, "General Astrophysics and Comparative Planetology with the Terrestrial Planet Finder Missions," JPL Publication 05-01 (2005)

3. W. Cash, "X-ray interferometry," Exp. Astr. 16, 91-139, (2003)

4. S. B. Shaklan, L. Marchen, J. J. Green and O. P. Lay, "The terrestrial planet finder coronagraph dynamics error budget" Proc. of SPIE 5905 to be published

5. LISA paper

6. M. Zhu and J. Hall, "Short and Long Term Stability of Optical Oscillators," Proc. IEEE Frequency Control Symposium, 44-55, (1992)

7. J. Ye, L. Robertsson, S. Picard, L.-S. Ma, and J. L. Hall, "Absolute frequency atlas of molecular $\mathrm{I}_{2}$ lines at $532 \mathrm{~nm}$," IEEE 48, 544-549 (1999)

8. J. L. Hall, L. S. Ma, M. Taubman, B. Tiemann, F. L. Hong, O. Pfister, and J. Ye, "Stabilization and frequency measurement of the $\mathrm{I}_{2}$-Stabilized Nd:YAG Laser," IEEE 48, 583-586 (1999)

9. G. Galzerano, C. Svelto, E. Bava, and F. Bertinetto, "High-frequency-stability diodepumped Nd:YAG lasers with the FM sidebands method and Doppler-free iodine lines at 532 nm," Appl. Opt. $386962-6966$ (1999)

10. S. Picard, L. Robertsson, L. S. Ma, K. Nyholm, M. Merimaa, T. E. Ahola, P. Balling, P. Křen, and J. P. Wallerand, "Comparison of ${ }^{127} \mathrm{I}_{2}$-stabilized frequency-doubled Nd:YAG lasers at the Bureau International des Poids et Mesures," Appl. Opt. 42, 1019-1028 (2003)

11. T. J. Quinn, "Practical realization of the definition of the metre, including recommended radiations of other optical frequency standards (2001)," Metrologia 40, 103-133 (2003) 
12. U. Roth, M. Tröbs, T. Graf, J. E. Balmer, and H. P. Weber, "Proton and gamma radiation tests on nonlinear crystals," Appl. Opt. 41 464-469 (2002)

13. J. L. Barth, M. Xapsos, and C. Poivey, "The radiation environment for the LISA/Laser interferometry space antenna," internal report (2005) 